\title{
Erratum to: Probing of Various Physiologically Relevant Metals-Amyloid- $\beta$ Peptide Interactions with a Lipid Membrane-Immobilized Protein Nanopore
}

\author{
Alina Asandei · Sorana Iftemi • Loredana Mereuta • \\ Irina Schiopu • Tudor Luchian
}

Published online: 30 April 2014

(C) Springer Science+Business Media New York 2014

\section{Erratum to: J Membrane Biol \\ DOI 10.1007/s00232-014-9662-z}

The article was published incorrectly due to an error in the publication process.

The colon (:) in between words 'metals' and 'amyloid$\beta$ ' was not in original submission, is introduced instead of '-' and therefore the title sounds altered. That is, the authors did not 'probe various physiologically relevant metals', as it may come out from the modified title; but 'probe various physiologically relevant metals-amyloid- $\beta$ peptide interactions'.

The correct title should be:

"Probing of Various Physiologically Relevant MetalsAmyloid- $\beta$ Peptide Interactions with a Lipid MembraneImmobilized Protein Nanopore"

The error in article title has been corrected with this erratum.

The online version of the original article can be found under doi:10.1007/s00232-014-9662-z.

\footnotetext{
A. Asandei - I. Schiopu

Department of Interdisciplinary Research, Alexandru Ioan Cuza

University, Blvd. Carol I, No. 11, 700506 Iasi, Romania

S. Iftemi $\cdot$ L. Mereuta $\cdot$ T. Luchian $(\bowtie)$

Laboratory of Molecular Biophysics and Medical Physics,

Department of Physics, Alexandru Ioan Cuza University, Blvd.

Carol I, No. 11, 700506 Iasi, Romania

e-mail: luchian@uaic.ro
} 